\title{
ON THE DYNAMICS \\ OF CONTRACTING RELATIONS
}

\author{
Vasile Glavan \\ Moldova State University \\ Chişinău, Republic of Moldova \\ University of Podlasie \\ Siedlce, Poland \\ glavan@usm.md vglavan@ap.siedlce.pl
}

\author{
Valeriu Guţu \\ Moldova State University \\ 60, A.Mateevici Str. \\ MD-2009 Chişinău, Republic of Moldova \\ gutu@usm.md
}

\begin{abstract}
Attractors of pointwise bounded and closed relations in metric spaces are considered. An analog of the asymptotic phase theorem for the contracting relations has been proved. The Shadowing Property near the attractor of a contracting relation is stated. As a consequence it is proved that the periodic points form a dense subset of the attractor.
\end{abstract}

Keywords: Relations, Attractors, Shadowing Property

\section{Introduction}

Dynamical systems have their roots in the pioneering works of Poincaré on the qualitative theory of differential equations. Discrete dynamical systems as iteration of diffeomorphisms appeared firstly as section maps of flows near periodic orbits. Later the concept evolved until the iteration of diffeomorphisms, or, recently, of noninvertible maps, became an independent subject of researches. More recently, iteration of finite collections of contractions, named as Iterated Function Systems (IFS), became the main tool for studying of the geometry of fractals as attractors, as well as the chaotic dynamics on these attractors. Until now the IFS remain one of the mostly elaborated multivalued dynamical systems.

The original version of this chapter was revised: The copyright line was incorrect. This has been corrected. The Erratum to this chapter is available at DOI: 10.1007/978-0-387-35690-7_44 
On the other hand, infinite dimensional dynamical systems, generated by partial differential equations without uniqueness (see [1], [2]), as well as control systems (see [3]), gave new motivations for studying the setvalued dynamics or, more precisely, the iterations of relations.

Evidently, such setting seems to be hopelessly general. However, it is demonstrated in [4] and [5] that the concept of limit sets, attractors, orbit and pseudo-orbit, transitivity and recurrence, known for ordinary flows, naturally generalizes for relations too (at least in the compact phase space setting).

The main goal of this paper consists in the developing of the notion of attractor for a relation in the noncompact setting, and in the studying of the dynamics near this attractor. One of the mostly used methods is the shadowing of pseudo-orbits. We give a criterion for a closed and bounded set to be the attractor of a contracting relation and prove the Shadowing Property of a such relation near the attractor. As a consequence, we obtain that the periodic points form a dense subset of the attractor.

\section{Dynamics of relations}

Let $(X, d)$ be a complete metric space. A relation on $X$ is a subset $f \subset X \times X$. Any relation can be regarded as a (set-valued) function from $X$ to the power set $\mathcal{P}(X)$, associating to each $x \in X$ a subset $f(x)$ of $X$. These two aspects of relations (set theoretical and functional) allows one to apply subset operations, such as union, intersection and closure, on the one hand, and the functional operations, such as composition, inverse, identity, on the other hand.

The neighborhoods of the diagonal as $V_{\varepsilon}=\left\{\left(x_{1}, x_{2}\right) \in X \times X\right.$ : $\left.d\left(x_{1}, x_{2}\right)<\varepsilon\right\}$, and similarly for $\overline{V_{\varepsilon}}$ with nonstrict inequality, are important examples of relations.

A relation on a metric space is said to be closed, if it is a closed subset of the Cartezian product of the space with itself. In a compact space this is equivalent to the upper semicontinuity of the relation (see [4]).

For two relations $f, g: X \rightarrow \mathcal{P}(X)$ we define the composition $g \circ f:$ $X \rightarrow \mathcal{P}(X)$ by: $(x, y) \in g \circ f$, if there exists $z \in X$ such that $(x, z) \in f$ and $(z, y) \in g$. The inverse of $f$ is, by definition, $f^{-1}:=\{(y, x)$ : $(x, y) \in f\}$. The composition is associative, so for $n \in \mathbf{N}$ we define $f^{n}$ to be the $n$-fold composition of $f$, and similarly $f^{-n}:=\left(f^{-1}\right)^{n}$. From associativity it follows that $f^{m+n}=f^{m} \circ f^{n}$ for $m, n \geq 0$ or $m, n \leq 0$. We call $\mathcal{O} f:=\bigcup_{n \geq 1} f^{n}$ the orbit relation.

A subset $A \subset X$ is called positive invariant with respect to a relation $f$ on $X$ (written " $A$ is $f$ tinvariant"), if $f[A] \subset A$, where $f[A]=\bigcup_{a \in A} f(a)$. 
Further, $A$ is called $f$ invariant, if $f[A]=A$. The last means that $A$ is $f$ +invariant and, in addition, $f^{-1}(x) \cap A \neq \emptyset$ for all $x \in A$.

For relations the analogous of the orbit is the notion of the chain. The finite or infinite sequence $\left\{x_{n}\right\} \subset X$ is called a chain for a relation $f$ : $X \rightarrow \mathcal{P}(X)$, if $x_{n+1} \in f\left(x_{n}\right)$ for all $n$, or, in other words, if $\left(x_{n}, x_{n+1}\right) \in f$.

Given a relation $f: X \rightarrow \mathcal{P}(X)$, a point $x \in X$ is called a fixed point for $f$, if $x \in f(x)$. Thus, $x \in \mathcal{O} f(x)$, if and only if there exists $n \geq 1$ such that $x \in f^{n}(x)$. Such point is called a periodic point for $f$.

Recall that for any two closed and bounded subsets $B_{1}$ and $B_{2}$ of a metric space $(X, d)$ the quantity $\varrho\left(B_{1}, B_{2}\right)$, given by

$$
\varrho\left(B_{1}, B_{2}\right):=\sup _{b_{1} \in B_{1}} \inf _{b_{2} \in B_{2}} d\left(b_{1}, b_{2}\right),
$$

defines the Hausdorff-Pompeiu metric as follows:

$$
H\left(B_{1}, B_{2}\right):=\max \left\{\varrho\left(B_{1}, B_{2}\right), \varrho\left(B_{2}, B_{1}\right)\right\} .
$$

This metric on the space $\mathcal{P}_{b, c l}(X)$ of all nonempty bounded and closed subsets of $X$ is complete, if $d$ is complete.

The following lemmas will be used in the next proofs.

Lemma 1.1. For any nonempty bounded subsets $B_{1}, B_{2} \subset X$ and $a$ point $x \in X$ the following inequality holds: $\varrho\left(x, B_{1}\right) \leq \varrho\left(x, B_{2}\right)+H\left(B_{1}, B_{2}\right)$. Proof. In the inequality $d\left(x, b_{1}\right) \leq d\left(x, b_{2}\right)+d\left(b_{1}, b_{2}\right),\left(x, b_{1}, b_{2} \in X\right)$, fix $b_{2} \in B_{2}$ and take infimum on $B_{1}$

$$
\inf _{b_{1} \in B_{1}} d\left(x, b_{1}\right) \leq d\left(x, b_{2}\right)+\inf _{b_{1} \in B_{1}} d\left(b_{1}, b_{2}\right) .
$$

The last term is not greater than $H\left(B_{1}, B_{2}\right)$. So we have for any $b_{2} \in B_{2}$ :

$$
\varrho\left(x, B_{1}\right) \leq d\left(x, b_{2}\right)+H\left(B_{1}, B_{2}\right) .
$$

Taking infimum on $B_{2}$, we obtain the desired inequality.

Lemma 1.2. Let $f: X \rightarrow \mathcal{P}_{b, c l}(X)$ be a relation such that

$$
H(f(x), f(y)) \leq \lambda d(x, y) \quad(x, y \in X)
$$

for some $\lambda>0$. Then $H\left(f\left[B_{1}\right], f\left[B_{2}\right]\right) \leq \lambda H\left(B_{1}, B_{2}\right)$ for any nonempty bounded subsets $B_{1}, B_{2} \subset X$.

Proof. Given any subsets $B_{1}, B_{2} \subset X$, we have

$$
H\left(f\left(b_{1}\right), f\left(b_{2}\right)\right) \leq \lambda d\left(b_{1}, b_{2}\right) \quad\left(b_{1} \in B_{1}, b_{2} \in B_{2}\right) .
$$

Passing consecutively to infimum on $B_{2}$ and supremum on $B_{1}$, we obtain $\varrho\left(f\left[B_{1}\right], f\left[B_{2}\right]\right) \leq \lambda \varrho\left(B_{1}, B_{2}\right)$. Analogously, $\varrho\left(f\left[B_{2}\right], f\left[B_{1}\right]\right) \leq \lambda \varrho\left(B_{2}, B_{1}\right)$. Thus, $H\left(f\left[B_{1}\right], f\left[B_{2}\right]\right) \leq \lambda H\left(B_{1}, B_{2}\right)$. 
Definition 1.1. A relation $f: X \rightarrow \mathcal{P}_{b, c l}(X)$ such that

$$
H(f(x), f(y)) \leq \lambda d(x, y) \quad(x, y \in X)
$$

for some $0<\lambda<1$ is called a contracting relation with the ratio $\lambda$.

Remark 1.1. Hyperbolic IFS and IFS with condensation (see [6]) consist one of the mostly developed examples of contracting relations.

\section{Attractors in relations}

The dynamics of a contracting mapping is trivial: the unique fixed point attracts all the points and even all bounded subsets of the phase space. As for set-valued functions the dynamics is much more complicated. Firstly, a fixed point, if it exists, need not be unique, nor attractive. Moreover, the set of fixed points is not even invariant. Secondly, the attractor of a hyperbolic IFS, a particular case of a contracting relation, is an invariant set, although containing the fixed points, it is a scene of a much more complicated dynamics, e.g. it contains a dense subset of periodic points as well as a dense chain [6].

There are various definitions of attractor. In ordinary dynamics (e.g. iterations of mappings) by an attractor one usually means an invariant set, which is dynamically indivisible and whose basin - the set of attracted points - is a large set. The dynamical indivisibility is understood as the existence of a dense orbit. As for the basin, it must contain a neighborhood of the attractor, or at least the nonvoid interior, sometimes positive Lebesgue measure is required. At the same time, relations need not be continuous or even semicontinuous, so for the basin to contain an (open) neighborhood is not an adequate demand. The "dynamical indivisibility" in the set-valued setting is also not very well understood.

In the case of compact spaces in [4] (see also [5]) the following definition has been proposed: $A$ is an attractor, if it is invariant and there exists a closed neighborhood $V$ of $A$ such that $\bigcap_{n \geq 0} f^{n}[V]$ is contained in $A$

In [1] and [2] the invariance $f[A]=A$ is relaxed up to the condition $f[A] \supset A$ with the assumption that $A$ attracts any bounded subset of a neighborhood of $A$.

Definition 2.1. $\quad$ Let $X$ be a metric space and $f: X \rightarrow \mathcal{P}(X)$ be a closed relation. A closed subset $A \subset X$ is called an attractor for $f$, if:

i) $f[A] \supset A$;

ii) there is a closed neighborhood $V$ of $A$ such that $\bigcap_{n \geq 0} f^{n}[V] \subset A$.

Remark 2.1. The second inclusion is, in fact, an equality. For, observe that $A \subset V$ and $A \subset f[A]$ imply the inclusions $A \subset f^{n}[V]$ for all $n \in \mathbf{N}$ and, hence, $A \subset \bigcap_{n \geq 0} f^{n}[V]$. 
Remark 2.2. Analogously, the first inclusion actually is an equality. For, use the second equality and the inclusions

$$
\bigcap_{n \geq 0} f^{n}[V]=A \subset f[A]=f\left(\bigcap_{n \geq 0} f^{n}[V]\right)=\bigcap_{n \geq 1} f^{n}[V] \subset A .
$$

Remark 2.3. For the "Cantor's relation" $f: \mathbf{R} \rightarrow \mathbf{R}, f(x)=\left\{\frac{1}{3} x\right\} \cup$ $\left\{\frac{1}{3} x+\frac{2}{3}\right\}$ the set of fixed points $A=\{0 ; 1\}$ satisfies the inclusion $A \subset$ $f[A]$, but the second condition does not hold.

Theorem 2.1. For any contracting relation on a complete metric space there exists an unique bounded and closed invariant set.

Proof. Let $f$ be a contracting relation on $X$. Denote by $f_{*}: \mathcal{P}_{b, c l}(X) \rightarrow$ $\mathcal{P}_{b, c l}(X), f_{*}(A)=f[A]$, the corresponding map on the complete metric space $\mathcal{P}_{b, c l}(X)$ of nonempty bounded and closed subsets of $X$, endowed with the Hausdorff-Pompeiu metric $H$. By Lemma 1.2, $f_{*}$ is a Lipschitz mapping, having the same contraction ratio as $f$. Hence, there exists an unique fixed point $A \in \mathcal{P}_{b, c l}(X)$. This means that the equation $f[A]=A$ has a solution in $\mathcal{P}_{b, c l}(X)$ and this solution is unique.

In the case of a hyperbolic IFS Barnsley [6] calls a set $A$, such that $f[A]=A$, "the attractor" for IFS. The following theorem shows that in the case of a contracting relation (and of a hyperbolic IFS, as a particular case) the Barnsley's notion of attractor coincides with Definition 2.1.

Theorem 2.2. $A$ bounded and closed subset $A \subset X$ is an attractor for a contracting relation $f$, if and only if $A$ is a compact invariant set for $f$.

Proof. If $A$ is a bounded attractor for $f$, then $A$ is a bounded invariant set for $f$ (see Remark 2.2). So $A$ is an unique fixed point for $f_{*}$ in the metric space $\mathcal{P}_{b, c l}(X)$. But we can repeat the proof of the Theorem 2.1 for the metric space $\mathcal{P}_{c p}(X)$ of nonempty compact subsets of $X$ and to obtain an unique solution $A \in \mathcal{P}_{c p}(X)$ of the equation $f[A]=A$. Since any compact subset is also bounded and closed, the bounded and closed invariant set, given by Theorem 2.1 , is actually compact.

Conversely, assume that $A$ is a compact, and so, a bounded and closed invariant set for $f$. Take a closed neighborhood $V$ of $A$ of small enough radius. It is bounded as well.

By Lemma 1.2, we have for any $n \in \mathbf{N}$

$$
\varrho\left(f^{n}[V], A\right)=\varrho\left(f^{n}[V], f^{n}[A]\right) \leq \lambda^{n} \varrho(V, A) .
$$

Because of the inclusion $f^{n+1}[V] \subset f^{n}[V]$, the last inequality implies:

$$
\varrho\left(\bigcap_{k=0}^{n} f^{k}[V], A\right) \leq \lambda^{n} \varrho(V, A) \quad(n \in \mathbf{N}) .
$$

Passing to the limit as $n \rightarrow \infty$, we obtain $\varrho\left(\bigcap_{k \geq 0} f^{k}[V], A\right)=0$, which is equivalent to the inclusion $\bigcap_{k \geq 0} f^{k}[V] \subset A$. Hence, $A$ is an attractor. 
Example. Let $\mathcal{C}[0,1]$ be the space of continuous functions on $[0,1]$ with the sup-metric. Fix $\psi \in \mathcal{C}[0,1]$ and consider the relation $f: \varphi \mapsto$ $\left\{\frac{1}{3} \varphi\right\} \cup\left\{\frac{1}{3} \varphi+\frac{2}{3} \psi\right\}$. Using Theorem 2.2, it is easy to check that the compact subset $K \subset \mathcal{C}[0,1], K=\{\tau \cdot \psi: \tau \in C\}$, where $C$ stands for the middle-third Cantor set, is the attractor of this relation.

Theorem 2.3. Let $f: X \rightarrow \mathcal{P}_{c p}(X)$ be a compact valued contracting relation with the ratio $0<\lambda<1$. Then for any chain $\left\{x_{n}\right\} \subset X$ and any $y_{0} \in X$ there exists a chain $\left\{y_{n}\right\} \subset X$, starting in $y_{0}$, such that

$$
d\left(x_{n}, y_{n}\right) \leq \lambda^{n} d\left(x_{0}, y_{0}\right) \quad(n \in \mathbf{N}) .
$$

Proof. It is known that for any nonempty compact subsets $A, B \subset X$ and any $a \in A$ there exists $b \in B$ such that $d(a, b) \leq H(A, B)$ (see, e.g. [7]). Therefore, for compact subsets $f\left(x_{0}\right), f\left(y_{0}\right) \subset X$ and given $x_{1} \in f\left(x_{0}\right)$ there exists $y_{1} \in f\left(y_{0}\right)$ such that

$$
d\left(x_{1}, y_{1}\right) \leq H\left(f\left(x_{0}\right), f\left(y_{0}\right)\right) \leq \lambda d\left(x_{0}, y_{0}\right) .
$$

Similarly, for given $x_{2} \in f\left(x_{1}\right)$ there exists $y_{2} \in f\left(y_{1}\right)$ such that

$$
d\left(x_{2}, y_{2}\right) \leq H\left(f\left(x_{1}\right), f\left(y_{1}\right)\right) \leq \lambda d\left(x_{1}, y_{1}\right) \leq \lambda^{2} d\left(x_{0}, y_{0}\right) .
$$

Moreover, inductively, for any $n \in \mathbf{N}$ there exists $y_{n} \in f\left(y_{n-1}\right)$ such that

$$
d\left(x_{n}, y_{n}\right) \leq \lambda^{n} d\left(x_{0}, y_{0}\right) .
$$

The chain $\left\{y_{n}\right\}$ is a required one.

As a corollary we obtain immediately the following result.

Theorem 2.4 (Asymptotic phase theorem for contracting relations). Let $f: X \rightarrow \mathcal{P}_{c p}(X)$ be a compact valued contracting relation with the ratio $0<\lambda<1$ and $A$ stand for the attractor of $f$. Then for any chain $\left\{x_{n}\right\} \subset X$ and any $a_{0} \in A$ there exists a chain $\left\{a_{n}\right\} \subset A$, starting in $a_{0}$, such that $d\left(x_{n}, a_{n}\right) \leq \lambda^{n} d\left(x_{0}, a_{0}\right)(n \in \mathbf{N})$.

\section{Skew-product relations}

This section is devoted to fiberwise contracting skew-product relations. We prove that any almost invariant cross-section can be approximated by an invariant one. This abstract setting will be applied in the next section for proving one of the main results of the paper - the Shadowing Property of contracting relations.

Given a relation $f: X \rightarrow \mathcal{P}(X)$, a set $Y$ and an injective mapping $\tau: Y \rightarrow Y$, let $F=(\tau, f): Y \times X \rightarrow \mathcal{P}(Y \times X)$ be the corresponding relation on $Y \times X$. With $p: Y \times X \rightarrow X$ for the projection, one has the following commutative diagram of relations: 


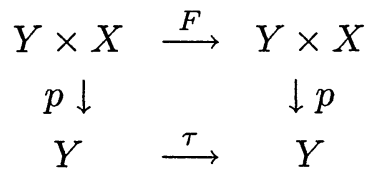

Commutativity means the equality $\tau \circ p=p \circ F$. A function $\sigma: Y \rightarrow$ $Y \times X$ such that $p \circ \sigma=\mathrm{id}_{Y}$ is called a cross-section. It is clear that any cross-section is of the form $y \mapsto(y, \varphi(y))$ for some function $\varphi: Y \rightarrow X$. In what follows we use the same notation for the cross-section and for this function.

Definition 3.1. One says that the cross-section $\sigma: Y \rightarrow Y \times X$ is $F$ + invariant, if $\sigma \circ \tau(y) \in F \circ \sigma(y)$ for any $y \in Y$.

Definition 3.2. Given $\varepsilon>0$, we call the cross-section $\sigma$ as $(F, \varepsilon)$ +invariant, if $\sigma \circ \tau(y) \in \overline{V_{\varepsilon}} \circ(F \circ \sigma(y))$, or, in other words, if $\varrho(\sigma \circ$ $\tau(y), F \circ \sigma(y)) \leq \varepsilon$ for any $y \in Y$.

Remark 3.1. If $f$ is pointwise closed and bounded, then the $(F, 0)$ tinvariance coincides with $F$ +invariance, which, in turn, means that for any $y \in Y$ the point $\sigma \circ \tau(y)$ belongs to the subset $F \circ \sigma(y)$. Similarly, the $(F, \varepsilon)$ +invariance means that the point $\sigma \circ \tau(y)$ is $\varepsilon$-close to the subset $F \circ \sigma(y)$, which, in turn, is equivalent to the +invariance with respect to the relation $\left(\tau, \overline{V_{\varepsilon}} \circ f\right): Y \times X \rightarrow \mathcal{P}(Y \times X)$.

Theorem 3.1. Let $(X, d)$ be a complete metric space and $f: X \rightarrow$ $\mathcal{P}_{b, c l}(X)$ be a relation on $X$. Let $Y$ be a set and $\tau: Y \rightarrow Y$ be an injection. Assume that $f$ is a contraction with ratio $0<\lambda<1$. Then for any $\varepsilon>0$ there is $\delta>0$ such that for any $(F, \delta)+$ invariant cross-section $\psi: Y \rightarrow Y \times X$ there exists a $F+$ invariant cross-section $\varphi: Y \rightarrow Y \times X$ such that $\bar{d}(\varphi, \psi):=\sup \{d(\varphi(y), \psi(y)): y \in Y\} \leq \varepsilon$.

Proof. Fix $\varepsilon>0$ and take $\delta=(1-\lambda) \varepsilon$. Let $\psi$ be any $(F, \delta)+$ invariant cross-section. Define $X_{\psi}$ as the space of all cross-sections $\alpha: Y \rightarrow Y \times X$ such that $\bar{d}(\psi, \alpha)<\infty$. It is easy to check that $\left(X_{\psi}, \bar{d}\right)$ is a complete metric space.

Let $X_{\psi, \varepsilon}$ denote the closed ball of radius $\varepsilon$ with center $\psi$ in $X_{\psi}$. Define a set-valued function (a relation) $\Phi$ on $X_{\psi, \varepsilon}$ as follows: $\Phi(\alpha)=$ $F \circ \alpha \circ \tau^{-1}\left(\alpha \in X_{\psi, \varepsilon}\right)$. Each value of this function is a closed and bounded subset of $X_{\psi}$. For, estimate

$$
\operatorname{diam} \Phi(\alpha) \leq \bar{H}(\Phi(\alpha), \Phi(\psi)) \leq \lambda \bar{d}(\alpha, \psi) \leq \lambda \varepsilon
$$

so $\Phi(\alpha)$ is a bounded set. Here $\bar{H}$ stands for the Hausdorff-Pompeiu metric on $\mathcal{P}_{b, c l}\left(X_{\psi}\right)$.

To prove the closedness of $\Phi(\alpha)$ take a convergent sequence $\left\{\xi_{n}\right\} \subset$ $\Phi(\alpha)$ with $\xi=\lim _{n \rightarrow \infty} \xi_{n}$. One has $\xi_{n}(y) \in f \circ \alpha \circ \tau^{-1}(y)(y \in Y)$. Since 
$f \circ \alpha \circ \tau^{-1}(y)$ is a closed subset of $X$, we obtain that $\xi(y) \in f \circ \alpha \circ \tau^{-1}(y)$ also, which implies $\xi \in \Phi(\alpha)$.

Moreover, for any $\alpha \in X_{\psi, \varepsilon}$ we have that $\Phi(\alpha) \cap X_{\psi, \varepsilon}$ is a closed nonempty subset of $X_{\psi, \varepsilon}$. To show this it is enough to state the inequality $\bar{\varrho}(\psi, \Phi(\alpha)) \leq \varepsilon(\bar{\varrho}$ is defined similarly as $\bar{H})$.

Fix $y \in Y$. For the taken value $\delta=(1-\lambda) \varepsilon$, using Lemma 1.1, we obtain:

$$
\begin{gathered}
\varrho\left(\psi(y), F \circ \alpha \circ \tau^{-1}(y)\right) \leq \varrho\left(\psi(y), F \circ \psi \circ \tau^{-1}(y)\right)+ \\
H\left(F \circ \psi \circ \tau^{-1}(y), F \circ \alpha \circ \tau^{-1}(y)\right) \leq \\
\delta+\lambda d\left(\psi \circ \tau^{-1}(y), \alpha \circ \tau^{-1}(y)\right) \leq \delta+\lambda \varepsilon=\varepsilon
\end{gathered}
$$

The right hand side does not depend on $y$, so $\sup _{y \in Y} \varrho\left(\psi(y), F \circ \alpha \circ \tau^{-1}(y)\right) \leq \varepsilon$, which implies that $\bar{\varrho}(\psi, \Phi(\alpha)) \leq \varepsilon$. Define a set-valued map $\Phi^{\prime}: X \rightarrow$ $\mathcal{P}_{c l}(X)$ as follows: $\Phi^{\prime}(\alpha)=\Phi(\alpha) \cap X_{\psi, \varepsilon}$. Since $\Phi^{\prime}(\alpha) \subset \Phi(\alpha)\left(\alpha \in X_{\psi, \varepsilon}\right)$, one has

$$
\bar{H}\left(\Phi^{\prime}(\alpha), \Phi^{\prime}(\beta)\right) \leq \bar{H}(\Phi(\alpha), \Phi(\beta)) \leq \lambda \bar{d}(\alpha, \beta),
$$

so $\Phi^{\prime}$ is a contracting set-valued mapping. By [8] (see also [7]), this mapping has at least one fixed point $\varphi \in X_{\psi, \varepsilon}$, i.e. $\varphi \in \Phi^{\prime}(\varphi) \subset \Phi(\varphi)$. The later means that $\varphi$ is an $F$ +invariant cross-section and $\bar{d}(\varphi, \psi) \leq \varepsilon$.

\section{Shadowing in contracting relations}

Definition 4.1. Given $\varepsilon>0$, a finite or infinite sequence $\left\{x_{n}\right\} \subset X$ is called an $\varepsilon$-chain for a relation $f: X \rightarrow \mathcal{P}(X)$ (or $(\varepsilon, f)$-chain, or pseudo-chain), if $\varrho\left(x_{n+1}, f\left(x_{n}\right)\right) \leq \varepsilon$ for all $n$.

Remark 4.1. One can easily see that a $(\varepsilon, f)$-chain is a chain for the relation $\overline{V_{\varepsilon}} \circ f$.

Thus, in addition to providing an abstract framework for generalizing properties of iterates of maps, the study of iteration of relations includes pseudo-chains ( $\varepsilon$-chains) as a special case of chains for a neighborhood $\overline{V_{\varepsilon}} \circ f$ of the relation $f$.

Definition 4.2. One says that the relation $f: X \rightarrow \mathcal{P}(X)$ has the Shadowing Property on the subset $A \subset X$, if, given $\varepsilon>0$, there exists $\delta>0$ such that for any $\delta$-chain $\left\{x_{n}\right\} \subset A$ there exists a chain $\left\{y_{n}\right\} \subset X$ such that $d\left(x_{n}, y_{n}\right) \leq \varepsilon$ for all $n$ (one says that the $\delta$-chain $\left\{x_{n}\right\}$ is $\varepsilon$-shadowed by the chain $\left.\left\{y_{n}\right\}\right)$.

Theorem 4.1. Any contracting relation $f: X \rightarrow \mathcal{P}_{b, c l}(X)$ has the Shadowing Property on $X$.

Proof. We will reduce the proof of this theorem to Theorem 3.1. For, take $Y=\mathbf{N}=\{1,2, \ldots\}$ and $\tau: \mathbf{N} \rightarrow \mathbf{N}, \tau(n)=n+1$. It is easy to see 
that the sequence $\left\{x_{n}\right\} \subset X, n \in \mathbf{N}$, is a chain (respectively, an $\varepsilon$-chain) for $f$, if and only if the function $\psi: \mathbf{N} \rightarrow \mathbf{N} \times X, \psi(n)=\left(n, x_{n}\right)$, is an invariant (respectively, an $\varepsilon$-invariant) cross-section for the relation $F=(\tau, f)$. Thus, the result follows from Theorem 3.1.

Corollary. If the relation $f$ on $X$ has the Shadowing Property on $X$, then for any $\varepsilon>0$ there exists $\delta>0$ such that for any relation $g$ on $X$, satisfying $H(f(x), g(x))<\delta(x \in X)$, any $\delta$-chain $\left\{x_{n}\right\}$ of $g$ is $\varepsilon$ shadowed by some chain $\left\{y_{n}\right\}$ of $f$.

Proof. It is known, that, given $\varepsilon>0$, there exists $\bar{\delta}>0$ such that any $\bar{\delta}$-chain $\left\{x_{n}\right\}$ of $f$ is $\varepsilon$-shadowed by some chain $\left\{y_{n}\right\}$ of $f$.

Take $\delta=\bar{\delta} / 2$. Let $g$ be a relation on $X$, satisfying $H(f(x), g(x))<$ $\delta(x \in X)$ and $\left\{x_{n}\right\}$ be a $\delta$-chain of $g$. At the same time, $\left\{x_{n}\right\}$ is a $(2 \delta)$-chain of $f$, since, by Lemma 1.1, we have

$$
\varrho\left(x_{n+1}, f\left(x_{n}\right)\right) \leq \varrho\left(x_{n+1}, g\left(x_{n}\right)\right)+H\left(g\left(x_{n}\right), f\left(x_{n}\right)\right)<2 \delta=\bar{\delta} .
$$

Hence, $\left\{x_{n}\right\}$, as a $\bar{\delta}$-chain of $f$, is $\varepsilon$-shadowed by some chain $\left\{y_{n}\right\}$ of $f$.

Remark 4.2. The Shadowing Property is not a robust property for the relations (even for contractions) in the sense that this property is to be kept under small perturbations of the relation with respect to the metric $\bar{H}$. For example, consider $f: \mathbf{R} \rightarrow \mathbf{R}, f(x)=\frac{1}{2} x$, and construct a closed relation $g$ on $\mathbf{R}$ as follows: $g$ is equal to $\mathrm{id}_{\bar{V}}$ on a small closed neighborhood $\bar{V}$ of zero, and to $f$ outside $\bar{V}$. The relation $g$ has not the Shadowing Property on $\mathbf{R}$.

Theorem 4.2. Let $f: X \rightarrow \mathcal{P}_{b, c l}(X)$ be a contracting relation. Then for any $\varepsilon>0$ there exists $\delta>0$ such that for any periodic $\delta$-chain $\left\{x_{n}\right\}$ there exists a periodic chain $\left\{y_{n}\right\}$ for $f$ such that $d\left(x_{n}, y_{n}\right) \leq \varepsilon$ for any $n \in \mathbf{N}$.

Proof. Fix $\varepsilon>0$ and take $\delta=(1-\lambda) \varepsilon$. Let $\left\{x_{n}\right\}$ be a periodic $\delta$ chain for $f$. Now apply Theorem 3.1 for $Y=\mathbf{Z}_{p}$ and $\tau: \mathbf{Z}_{p} \rightarrow \mathbf{Z}_{p}$, $\tau(n) \equiv n+1(\bmod p)$. Thus, we obtain a $p$-periodic chain $\left\{y_{n}\right\}$, which $\varepsilon$-shadows $\left\{x_{n}\right\}$.

Remark 4.3. It is obvious that every periodic chain for a contracting relation is contained in the attractor. From Theorem 4.2 it follows that any periodic $\delta$-chain must be in a $\varepsilon$-neighborhood of the attractor, since $\varrho\left(x_{n}, A\right) \leq d\left(x_{n}, y_{n}\right)$ for any $n \in \mathbf{N}$.

Recall that $x \in X$ is named a periodic point for the relation $f: X \rightarrow$ $\mathcal{P}(X)$, if there exists a periodic chain, starting at $x$, or, in other words, if $x$ is a fixed point for the orbit relation $\mathcal{O} f=\bigcup_{n \geq 1} f^{n}$. Following [4], denote by $|\mathcal{O} f|$ the set of periodic points of $f$. 
Theorem 4.3. For a contracting relation $f: X \rightarrow \mathcal{P}(X)$ the periodic points form a dense subset of the attractor, i.e. $\overline{|\mathcal{O} f|}=A$.

Proof. For any $\varepsilon>0$ and any point $x \in A$ we have to find a periodic point $y \in A$ such that $d(x, y)<\varepsilon$. If $x$ is a periodic point it is nothing to prove.

Assume that $x$ is not a periodic point, i.e. $x \notin f^{n}(x)$ for any $n \in \mathbf{N}$. Because of compactness of $A$ for any $\eta>0$ there exists $n$ such that $\varrho\left(x, f^{n}(x)\right) \leq \eta$. For $\eta<(1-\lambda) \varepsilon / 2$ take a (finite) chain $x_{1}=$ $x, x_{2}, \ldots, x_{n} \in f^{n}(x)$ such that $d\left(x, x_{n}\right) \leq \eta$ and extend it up to a periodic $\eta$-chain $\left\{x_{1}, x_{2}, \ldots, x_{n}, x_{n+1}=x_{1}, \ldots\right\}$. In virtue of Theorem 4.2 for this periodic $\eta$-chain there exists a periodic chain $\left\{y_{n}\right\}$ such that $d\left(x_{n}, y_{n}\right) \leq \varepsilon / 2<\varepsilon$ for any $n \in \mathbf{N}$. Thus, $y=y_{1}$ is the desired periodic point.

Theorem 4.4. Let $f: X \rightarrow \mathcal{P}_{b, c l}(X)$ be a contracting relation and $A$ stand for its attractor. Then for any $a \in A$ one has $\overline{\mathcal{O} f(a)}=A$.

Proof. Because of $\mathcal{O} f=\bigcup_{n \geq 1} f^{n}$, the inclusion $\overline{\mathcal{O} f(a)} \subset A$ follows from the invariance of $A$ and its closedness.

For proving the inverse inclusion we use the Lemma 1.2 and observe that $H\left(f^{n}(a), A\right)=H\left(f^{n}(a), f^{n}[A]\right) \leq \lambda^{n} H(a, A) \rightarrow 0$ as $n \rightarrow \infty$.

Thus, for any $b \in A$ and any $\varepsilon>0$ there exists natural $n$ such that $\varrho\left(b, f^{n}(a)\right)<\varepsilon$.

Due to the compactness of $A$ we have that for any $b \in A$ and any $\varepsilon>0$ there exist natural $n$ and $a^{\prime} \in f^{n}(a)$ such that $d\left(b, a^{\prime}\right)<\varepsilon$. This implies the equality $\overline{\mathcal{O} f(a)}=A$.

\section{References}

[1] Valero J. Attractors of parabolic equations without uniqueness. J. Dynam. Diff. Eq., Vol. 13, 4(2001), 711-744.

[2] Melnik V.S, Valero J. On attractors of multivalued semi-flows and differential inclusions. Set-valued Analysis, 6(1998), 83-111.

[3] Bobylev N., Emelianov S., Korovin S. Attractors in control systems. Different. Uravnenya, 35(1999), 617-622.

[4] Akin E. The General Topology of Dynamical Systems. Amer. Math. Soc., Providence, RI, 1993.

[5] McGehee R. Attractors for closed relations on compact Hausdorff spaces. Indiana U. Math. J., Vol. 41, 4(1992), 1165-1209.

[6] Barnsley M. Fractals Everywhere. Academic Press Professional, Boston, 1988.

[7] Rus I.A. Generalized Contractions and Applications. Cluj University Press, ClujNapoca, 2001.

[8] Nadler S.B. Multi-valued contraction mappings. Pacific J. Math., 30(1969), 475488. 